\title{
Finding the Optimized Frequency of Electric Field on the Attempt of Reducing Blood Sugar Level in Type II Diabetes Patients
}

\section{Suhariningsih¹, Dwi Winarni², Saikhu Akhmad Husen², and Tri Anggono Priyo ${ }^{3}$}

${ }^{1}$ Department of Health, Vocational Faculty, Universitas Airlangga; Department of Physics, Science and Technology Faculty, Universitas Airlangga, Campus C, Jl. Mulyorejo, Surabaya, Indonesia, 60115

${ }^{2}$ Department of Biology, Science and Technology Faculty, Universitas Airlangga, Campus C, J. Mulyorejo, Surabaya, Indonesia, 60115

${ }^{3}$ Department of Physics, Science and Technology Faculty, Universitas Airlangga, Campus C, Jl. Mulyorejo, Surabaya, Indonesia, 60115

\section{Abstract}

A study has been conducted to test the use of electric fields to lower blood sugar levels

Corresponding Author:

Suhariningsih

suhariningsih.unair@gmail.com

Received: 8 June 2018

Accepted: 17 July 2018

Published: 8 August 2018

Publishing services provided by

Knowledge $\mathrm{E}$

(c) Suhariningsih et al. This article is distributed under the terms of the Creative Commons Attribution License, which permits unrestricted use and redistribution provided that the original author and source are credited.

Selection and Peer-review under the responsibility of the 2 nd ICVHE Conference Committee. on rats with type II Diabetes Mellitus. This study uses male rats (Rattus novergicus), aged 8-12 weeks weighing 150-200 grams, with a total of 31 rats. Therapy was conducted by putting rats in a chamber that emits an electric field every day for 1 hour for 28 days. To search for the optimum frequency of the treatment, frequency variations of $15,30,60$, and $120 \mathrm{kHz}$ were given. The electrical charge distribution of the air in the room was then measured, whereas mice blood sugar levels were also measured to reveal the effect of the treatment on cell tolerance to sugar, and to come up with the measurement of insulin levels at the end of the treatment. The results of measurements of the electrical charges in the air were very significant generally showing that the treatment with a frequency of 15 to $120 \mathrm{KHz}$ for four weeks in the experimental animals could lower their blood sugar (fasting period), but the success rate significantly occurred at a frequency of $15 \mathrm{kHz}$. The decrease in fasting blood sugar levels after the treatment was probably due to the influence of the treatment (the influence of an electric field) that improved the regulation of insulin receptors. Improvements in the regulation of insulin receptor activities with high gluconeogenesis may be the cause of a decrease in fasting blood sugar levels that is not accompanied by improved cell tolerance to sugar.

Keywords: electric field, blood sugar levels, insulin levels

\section{Introduction}

The purpose of this research is to find out the optimum value of the electric charge G OPEN ACCESS frequencies that can lower the blood sugar level of type II diabetes mellitus patients. 
To seek for the optimum frequency in therapy, the frequencies chosen were 15,30 , 60 , and $120 \mathrm{kHz}$. Air electric charge measurements were carried out to find out if the electric field was physiologically working. As samples of therapy in this research, 31 male rats (Rattus novergicus) aged between 8 and 12 weeks weighing 150 and $200 \mathrm{mgs}$ were chosen.

Electric field charges were studied to be able to give positive effects on tested animals at physical, cellular, and physiological levels [5]. The biophysical review of this research is as follows: First, electric field radiation can trigger air ionization which causes the air to contain positive and negative (electrons) electric charges, while the released electrons can join oxygen to create ozone $\left(\mathrm{O}_{3}\right)$. Electrons and ozone produced by electric field are very beneficial if inhaled because they neutralize poisons in the body. Second, blood contains both negative and positive ions $\left(\mathrm{Fe}^{+2}, \mathrm{Fe}^{+3}, \mathrm{Mg}^{+}, \mathrm{Cl}^{-}\right.$, etc.), and with the presence of electric field, an electronic polarization can occur [1] in which the positive ions will move in the same direction with electric field and the negative ions will move on the opposite direction. Electric movement will then occur which causes bias index changes [4], and an increase in membrane permeability, causing an increase in an influx of water and ions inside the membrane [7]. Third, if the electric field is an alternating one, there is a high possibility of collision among the ions which can cause vasodilatation in blood vessels. According to Jiang et.al (1997), electric field can create a vasodilatation effect on blood vessels followed by the release of endothelium-derived nitrite oxide that can cause the relaxation of smooth muscles in the walls of blood vessels. Blood vessel dilatation can speed up the flow of blood and this increase in speed can lead to the decreasing blood viscosity.

Diabetes mellitus type 2 (DM 2 ) is characterized by the insulin resistance in insulinsensitive cells. These insulin-sensitive cells (skeletal and cardiac muscle cells, hepatocytes, and fatty cells/adipocytes) have an insulin receptor (IR) on their surfaces. Insulin resistance can be caused by mutation or the loss of expressions in the enzymes involved in the insulin signaling line.

In a normal condition, insulin will be secreted by the pancreas beta cells if there is an increase in the blood glucose level. The secreted insulin will be released into the blood vessels and will bond with IR in the cell membranes which are sensitive to the insulin. The bond between insulin and IR will cause the autophosphorylation of tyrosine (the addition of phosphate clusters in IR tyrosine). This activation causes the phosphorylation of tyrosine in IRS1 (insulin receptor-substrate). The phosphorylated IRS1 then binds $\mathrm{Pl}_{3} \mathrm{~K}$ (phosphoinositide -3-kinase) which will experience translocation to the membrane surface where $\mathrm{Pl} 3 \mathrm{~K}$ will convert phosphatidylinositol $(4,5)$ phosphate 
$(\mathrm{PI}(4,5) \mathrm{P} 2$ into phosphatidylinositol $(3,4,5)$ phosphate $(\mathrm{PI}(3,4,5) \mathrm{P}$. This phosphorylation causes further phosphorylation in the AKT protein. The activation of the AKT protein and $\mathrm{PI}_{3} \mid \mathrm{K}$ results in the translocation of GLUT4 (the protein receptor for glucose) onto the membrane surface (Figure 1). With the exposure of GLUT4, there is an increase in the glucose transport. The increase in the glucose transport in cells will lower the level of blood glucose. The increase in AKT 4 and the increase in the glucose level in cells will increase the activity of glycogen synthesis.

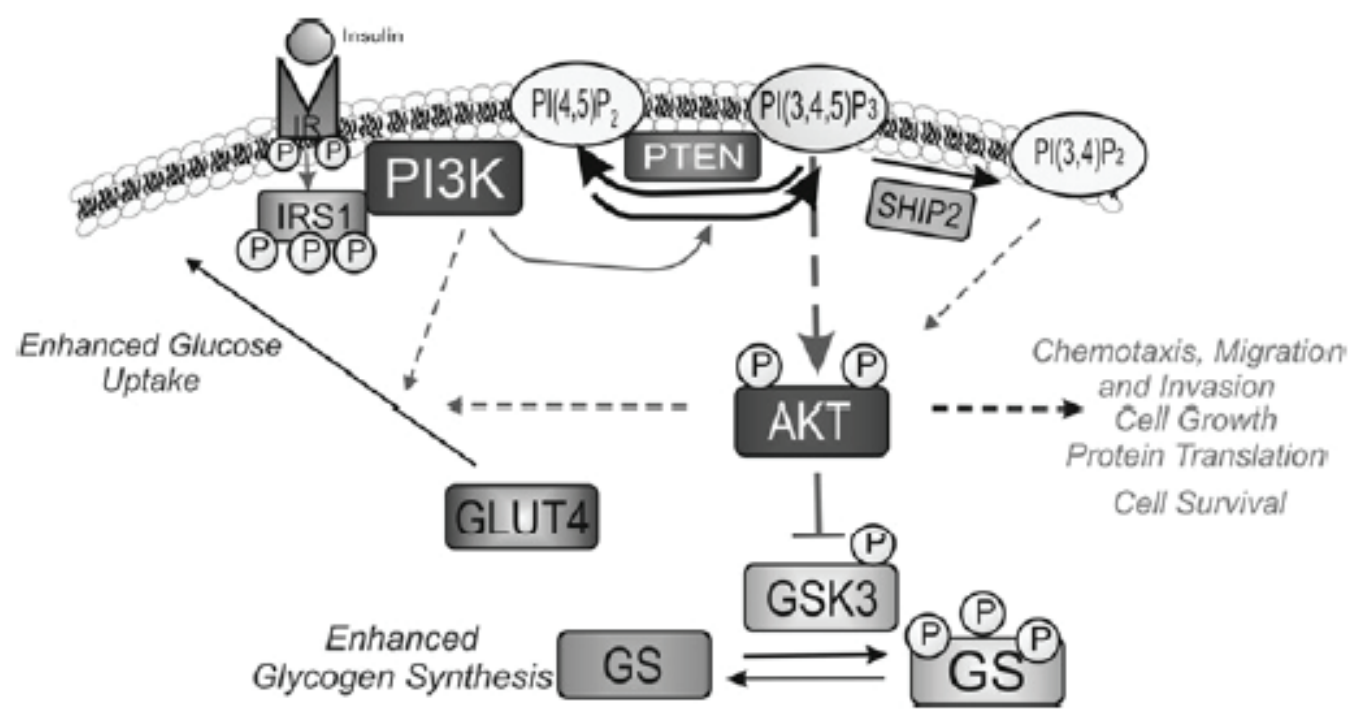

Figure 1: Insulin signaling line. One of the effects of the bonding of insulin with its receptor (IR) is the translocation of GLUT4 (receptor for glucose) on the cell membrane surface that will increase the speed of glucose transportation in the cell.

DM 2 condition can be induced experimentally just like the induction carried out on test animals used in this research - by using streptozin (STZ) and nicotinamide. The use of STZ increases the level of reactive oxygen species (ROS) in the circulation. The increasing ROS level will activate the phosphorylation of serine/threonine in IRS-1. This phosphorylation increases the speed of IRS-1 degradation. The decreasing quantity of IRS-1 obstructs the formation of phosphatidyl inositol $(4,5)$ phosphate ( $\mathrm{PI}(4,5) \mathrm{P} 2$ to become phosphatidyl inositol $(3,4,5)$ phosphate $\left(\mathrm{PI}(3,4,5) \mathrm{P}_{3}\right.$. This obstruction also causes the obstruction the phosphorylation of AKT protein which eventually will hamper the translocation of GLUT 4 onto the cell surface. The decreasing quantity of GLUT 4 which is exposed on the cell surface decreases the transportation speed of glucose in the cell. This speed decrease raises the blood sugar level. Nicotinamide is known to be able to obstruct the formation of ROS. Nicotinamide is used to prevent further destruction of the pancreas caused by DM 1. 
The decreasing supply of glucose into cells causes the conversion of fatty acid from the fatty cells (the result of lipolysis) and amino acid from muscles (the result of muscle protein breakdown) to become glucose in the liver cells through gluconeogenesis.

\section{Experimental Method}

The metal raster is functioned as an antenna which is put under the mattress, and connected with an alternating current electric generator with the frequencies of 15 , 30,60 , and $120 \mathrm{kHz}$. When the electric field is activated, there is a significant change in the value of the air capacitance above the mattress, which shows that there is a change in the constant of the air dielectrics. The interaction between the electric field and air is observed with an aligned plate capacitance meter placed above the mattress (the air is functioned as a dielectric material). The change in the air composition caused by electric field shows that the electric field has worked and can be used to treat the animals with DM 2.

The animals used in this research were 31 male rats (Rattus novergicus) aged 8-12 weeks weighing 8-12 weeks. Before randomly divided into 8 groups in which 4 were normal groups (K-15, K-30, K-60, and K-120, without STZ induction and nicotinamide) and 4 DM groups (D-15, D-30, D-60, and D-120, induced with STZ and nicotinamide), all the rats were checked for their fasting blood glucose level (blood glucose level without calorie intake for 12 hours) to insure that all of them had normal blood glucose level of $<126 \mathrm{mg} / \mathrm{dl}$ (Barik et al., 2008). Each group consisted of 4 rats. Therapies to all groups were carried out by placing the rat groups on the mattress for one hour every day for 4 weeks (28 days), with a frequency variations of $15 \mathrm{kHz}$ (for one normal group and one DM group), $30 \mathrm{kHz}$ (for one normal group and one DM group), $60 \mathrm{kHz}$ (for one normal group and one DM group), and $12 \mathrm{kHz}$ gfor one normal group and one DM group). Throughout the therapy, there was a measurement of the changes of the air electric charge distribution above the mattress, using a capacitance meter. Once a week during the therapy, each rat was checked for its fasting blood glucose level (blood glucose level without calorie intake for 12 hours). The blood was taken from the rats' tail vena which was then analyzed using a glucometer (Accu-Check, Roche Diagnostic, USA). After 4 weeks of therapy, the rats' blood was taken in an intra cardiac way to be further analyzed with rat insulin ELISA kits.

\section{Result}




\subsection{The measurement of electric charge distribution above the mattress}

TABLE 1: Instrument used: A digital capacitance meter that has 3 measurement ranges, which are $1 \mathrm{pF}$ precision (used in this experiment), $1 \mathrm{nF}$ precision, and $1 \mu \mathrm{F}$ precision.

\begin{tabular}{|c|c|c|}
\hline No & Frequency $(\mathrm{kHz})$ & Capacitance $(\mathrm{pF})$ \\
\hline 1 & $\star$ & 71 \\
\hline 15 & 252 \\
\hline 30 & 208 \\
\hline 40 & 135 \\
\hline 60 & 111 \\
\hline 80 & 69 \\
\hline 120 & 52 \\
\hline 160 & 33 \\
\hline Note: ${ }^{*}$ ) without electric field & \\
\hline
\end{tabular}

Based on the observation results, it can be concluded that the highest capacitance value is the electric field exposure at the frequency of $15 \mathrm{kHz}$. The higher the frequency of the electric field generator $(30,60,120 \mathrm{kHz})$, the lower the capacitance value, which means that there is no change in the electric charge in the air.

\subsection{The influence of treatments on fasting blood sugar level}

The fasting blood sugar levels during the 28-day treatment (blood sugar level observation was carried out in an interval of 7 days), with a treatment duration of 1 hour per day on the rats, can be seen in Picture 2 . The statistical test results with one variant analyses, or the Brown-Forsythe test, followed by the Duncan test, or $t$-test for the 2 free samples with $\nabla=0.05$ in each blood sugar measurement time (the beginning of treatment, weeks 1, 2, 3, and 4) showed that the treatments could lower the blood sugar levels (fasting).

Significant decreases occurred to the groups that were given the $15 \mathrm{kHz}$ treatment (all samples show a decrease). The fasting blood sugar levels in the beginning of treatment which were categorized into mild to medium-level diabetes (blood sugar level until 250) could drop to normal blood sugar levels. Meanwhile, those categorized as heavy DM (above $300 \mathrm{~g} / \mathrm{dl}$ ) experienced drops from normal to mild levels. The 
same condition also happened to the groups that received the $30,60,90$, and $120 \mathrm{KHz}$ treatments

Several data that need attention are:

1. Heavy DM was found in the $30 \mathrm{kHz}$ group ( 1 from 4 samples) in which originally it was categorized as heavy DM (fasting blood sugar level $>400 \mathrm{~g} / \mathrm{dl}$, in week 1 it changed to mild, the becoming normal in week 2, but returning back to heavy DM (blood sugar level > 300) in week 3 until the end of treatment even though the fasting blood sugar level showed a decrease if compared to that in the beginning of treatment.

2. In the $60 \mathrm{kHz}$ group, the decrease in blood sugar levels in all samples did not reach the normal blood sugar level. Nonetheless, from the observation, it was found that medium DM became heavy one (1 out of 4 samples), and mild DM became a heavy one ( 1 out of 4 samples).

3. In the $120 \mathrm{kHz}$ group, one out of 4 samples changed from medium DM to a heavy one at the end of treatment.
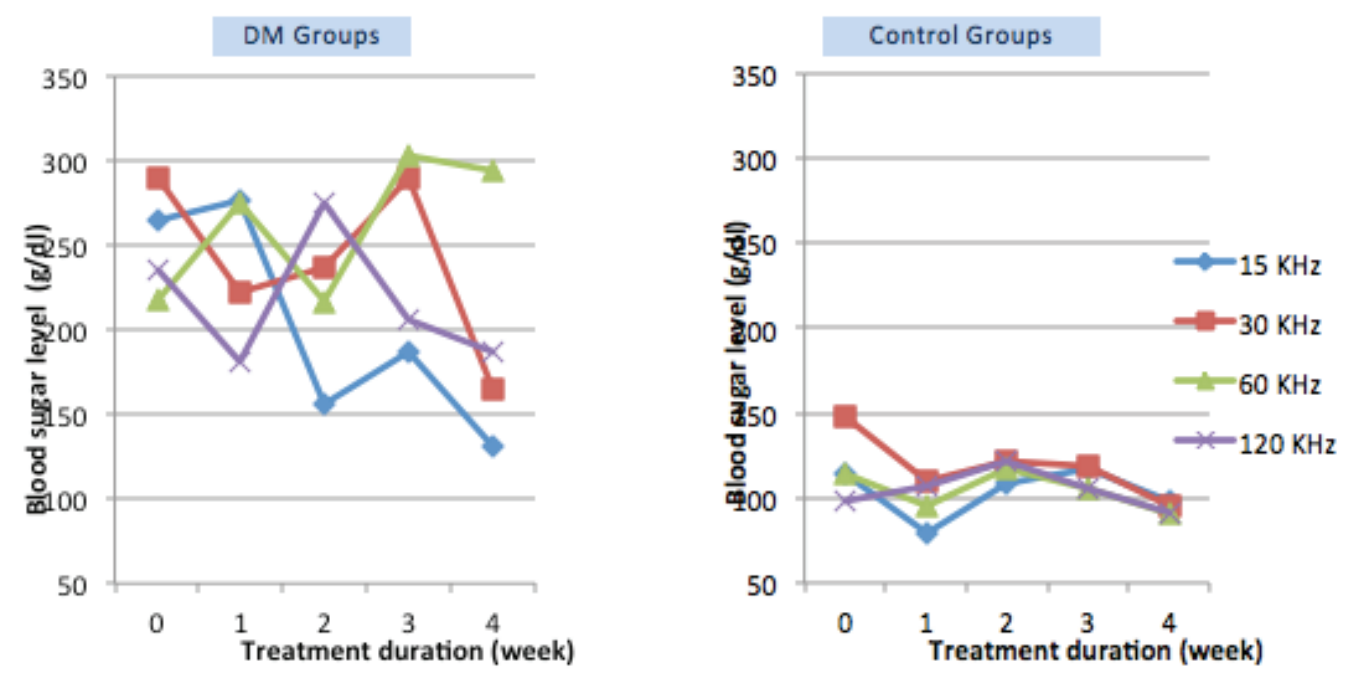

Figure 2: Means of blood sugar level changes during the treatment on the mattress for the DM group and normal group (control group).

Based on the aforementioned statistical test results, it can be summed up that, in general, treatments with frequencies from $15 \mathrm{kHz}$ to $120 \mathrm{kHz}$ for 4 weeks to the tested animals could lower blood sugar levels (fasting), but the level of success occurred significantly at the frequency of $15 \mathrm{kHz}$, while for the frequencies of 30,60, and 120 $\mathrm{kHz}$, the level of success still needed re-examination. 
The duration of treatment also needed further examination. For example, with the treatment using the $15 \mathrm{kHz}$ frequency for 4 weeks for heavy DM, even though there were decreases to the normal level, there was still a mild DM case.

\subsection{The effects of treatment on cell tolerance toward sugar}

Glucose tolerance test (GTT) was conducted by giving D-glucose $2 \mathrm{~g} / \mathrm{kg}$ solution orally to the rats which had fasted the night before. The blood sugar level was then measured at minutes $0,30,60,90$, and 120. In a normal condition, the blood sugar level will rise after being given $\mathrm{D}$-glucose, then will drop back to the normal level range (less than $200 \mathrm{~g} / \mathrm{dl})$.

Rats from the result of streptozotocin (STZ) induction were categorized to be diabetic if their fasting blood sugar level > $126 \mathrm{~g} / \mathrm{dl}$ and/or the blood sugar level during GTT did not show a decrease to normal levels until the end of GTT. The changes in blood sugar levels during GTT before and after the mattress treatment are indicated in Figure 2. The data were analyzed using one-way variant analysis or Kruskall Wallis, continued with the Duncan test or Mann Whitney at $\mathbb{Q}=0.05$.

The result of data analysis showed that at the beginning of the treatment, there were no significant differences in cell tolerance to glucose between the DM groups (except in minute 90 in the $15 \mathrm{kHz}$ group) and the control groups. Differences in the changes of blood sugar levels between the DM patients and the normal ones could be seen from by significant changes in blood sugar levels during GTT in the DM group against those of the control group in all measurement times (30,60, 90, and 120 minutes after D-glucose giving).

The mattress treatment for a month that showed decreases in fasting blood sugar levels turned out to not be followed by significant changes in the cell tolerance toward sugar. Table 1 shows the data statistical test results of blood sugar levels during GTT which was carried out at the end of treatment.

The analysis results showed that after glucose was given, the cell tolerance toward glucose was different significantly in the normal group, with higher levels in each observation time.

\subsection{Insulin levels at the end of treatment}

The measurement of insulin levels at the end of treatment can be used to detect if the pancreas is still functioning normally or not in synthesizing insulin. Insulin is measured 
TABLE 2: The statistical test results of one-way variant analysis or Kruskall Wallis, followed by Duncan test or Mann Whitney at $\otimes=0.05$ with the blood sugar level data during GTT (glucose tolerance test) (DM = diabetes mellitus).

\begin{tabular}{|c|c|c|c|c|}
\hline \multicolumn{5}{|c|}{ The results of blood sugar level analysis during GTT in minute: } \\
\hline 0 & 30 & 60 & 90 & 120 \\
\hline $\begin{array}{l}\text { No significant } \\
\text { differences } \\
\text { among DM } \\
\text { groups, among } \\
\text { control groups, } \\
\text { and between DM } \\
\text { and control } \\
\text { groups. }\end{array}$ & $\begin{array}{l}\text { No significant } \\
\text { difference } \\
\text { between DM } \\
\text { among DM group, } \\
\text { and among } \\
\text { control groups, } \\
\text { Significant } \\
\text { difference } \\
\text { between DM and } \\
\text { control groups }\end{array}$ & $\begin{array}{l}\text { No significant } \\
\text { difference among } \\
\text { DM groups } \\
\text { (except } 60 \mathrm{KHz} \\
\text { with } 120 \mathrm{KHz} \text { ) } \\
\text { Significant } \\
\text { difference } \\
\text { between DM and } \\
\text { control groups }\end{array}$ & $\begin{array}{l}\text { No significant } \\
\text { difference among } \\
\text { DM groups } \\
\text { Significant } \\
\text { difference } \\
\text { between DM and } \\
\text { control groups }\end{array}$ & $\begin{array}{l}\text { No significant } \\
\text { difference among } \\
\text { DM groups } \\
\text { Significant } \\
\text { difference } \\
\text { between DM and } \\
\text { control groups }\end{array}$ \\
\hline
\end{tabular}

\section{DM Group}

Before treatment

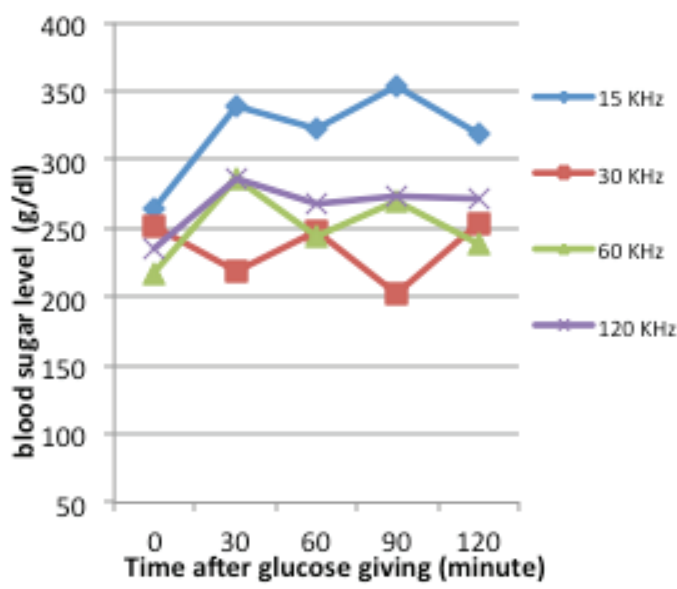

After treatment

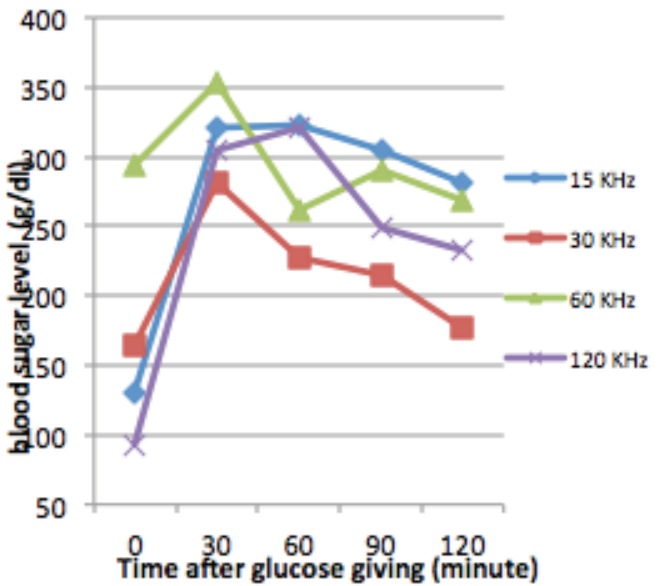

Figure 3: Means of changes in blood sugar levels during glucose tolerance test on diabetic rats induced with STZ (DM groups), before and after treatments.

using Rat Insulin ELISA Kit (Crystal Chem). The means of the measurement results can be seen in Figure 5 . The results of the data analysis with Brown Forsythe test at $\nabla$ $=0.05$, showed that there was no significant difference between the level of insulin among the DM grouups, among the control groups, and between control groups and DM groups. As a result, the prolonged DM condition until the end of treatment in some samples as mentioned in sub chapter 1 did not result in the decreasing function of the pancreatic beta cells in producing insulin.

Insulin secreted by the pancreatic beta cells will bind with specific receptors located on the target cell membranes. The binding of insulin with the target cell results in 

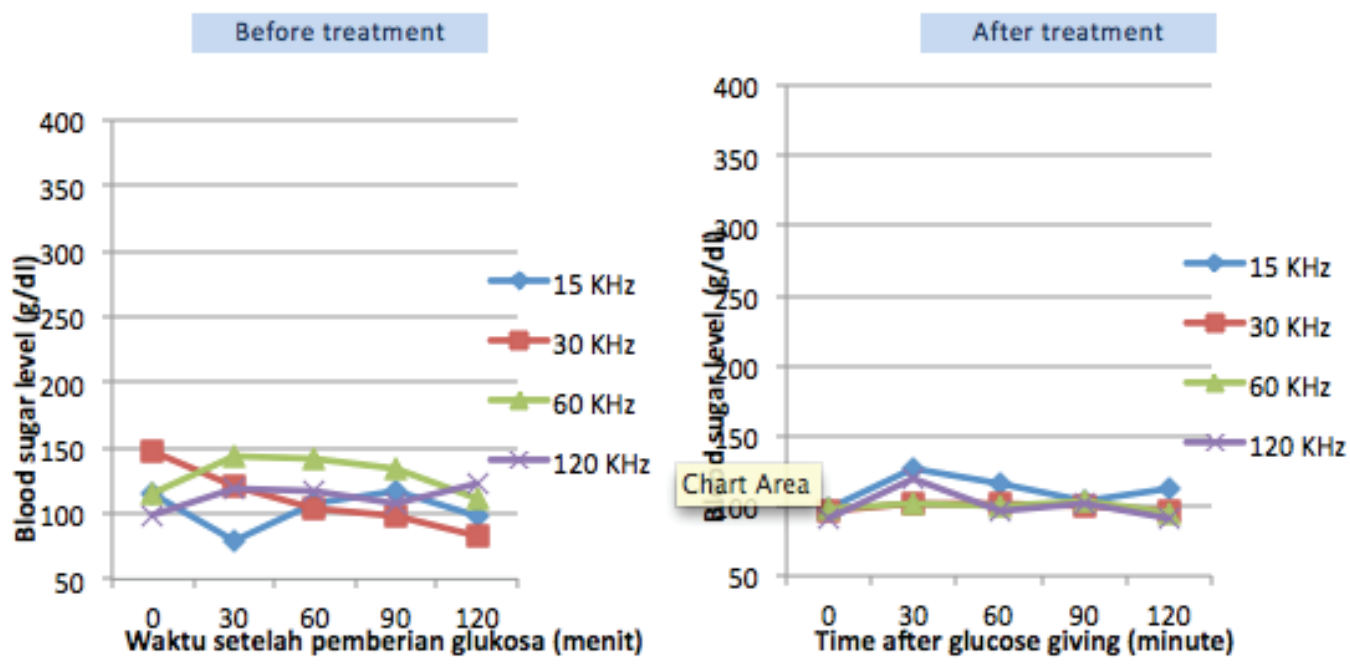

Figure 4: Means of changes in blood sugar levels during glucose tolerance test on diabetic rats induced with STZ on rats with normal blood sugar levels (control groups) before and after treatments.

the exposure of sugar transporting protein which relies on insulin (GLUT-4) on the cell surface. The exposed GLUT-4 will then fasten the speed of sugar intake by cells. GLUT-4 protein is found in cardiac muscles, skeletal muscles, and fatty cells in adipose tissues.

Diabetes mellitus (DM) is caused by the decrease in sugar uptake by the peripheral tissues and the increase of gluconeogenesis. Sugar uptake decrease and gluconeogenesis increase in DM type-1 patients are caused by the low level of insulin due to the damage of pancreatic beta cells, while in DM type 2 patients it is caused by cell resistance against insulin which is caused the down regulation of insulin receptor which eventually decreases the number of GLUT-4 in cell surfaces.

Gluconeogenesis is the glucose synthesis from non-carbohydrate raw materials carried out by the liver cells. The increase in gluconeogenesis in DM patients require raw materials which come from the increasing breakdown of lipid fatty cells and protein in muscle cells. The lipid breakdown and the mobilization of the breakdown results in the circulation will rise which will influence the plasma lipid profiles. The increasing lipid breakdown will also increase the plasma viscosity.

The insulin levels which are not significantly different with the normal groups indicate that there is a contribution of repair in the insulin receptor regulation which has a role in decreasing the blood sugar level. This indirectly is connected also with the improvement of GLUT-4 exposure on the surface. The decrease in the fasting blood 


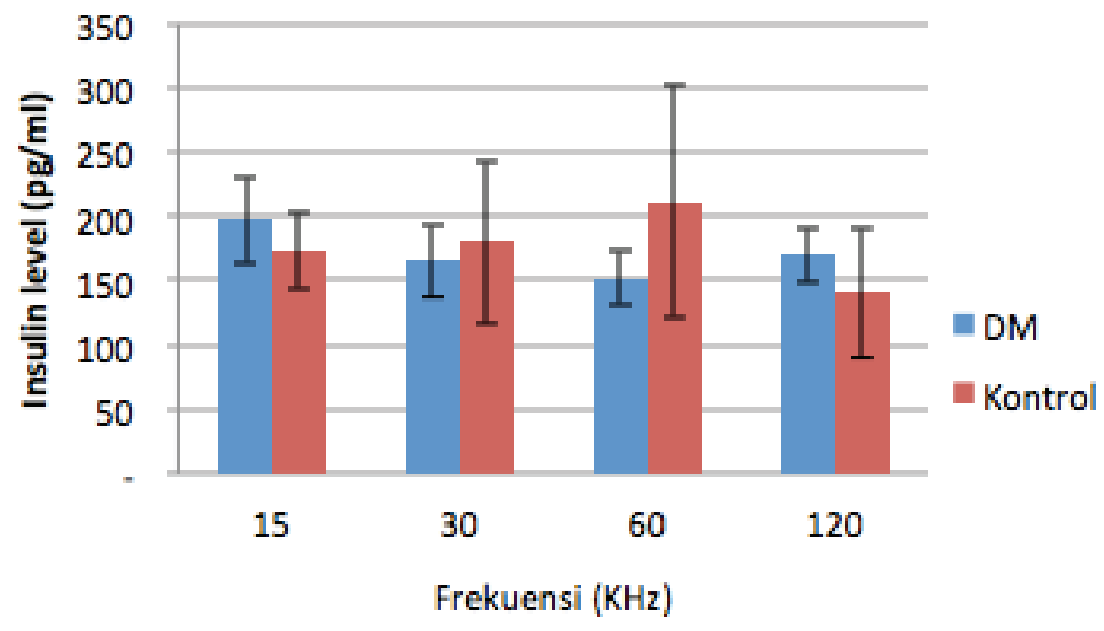

Figure 5: Means of insulin levels after treatment.

sugar level during the mattress treatment is probably due to the influence of treatment in restoring the insulin receptor regulation. This insulin receptor regulation improvement during a still high gluconeogenesis activity can cause the decrease in fasting blood sugar levels which are not followed by the improvement of cell tolerance toward sugar. To acquire more in-depth answers, there should be a measurement/research about the activities of gluconeogenesis such as the plasma lipid profile and the glucose liver functions (not carried out in this research).

\section{Conclusions}

1. The highest capacitance value was the electric field exposure at the frequency of $15 \mathrm{kHz}$.

2. The level of success in the DM II therapy happened significantly at the $15 \mathrm{kHz}$ frequency where in all samples there was a decrease.

3. Treatment for a month that showed the decreasing blood sugar levels turned out not to be followed by a significant decrease in cell tolerance toward sugar. This is due to the duration of treatment which was not long enough (only 28 days).

4. The decrease in blood sugar levels after treatment was probably caused by the improvement in the insulin receptor regulation. Improvement in the insulin receptor regulation with high gluconeogenesis activities can be the cause of the decrease in fasting blood sugar levels which was not followed by improvement 
in the cell tolerance toward sugar. To gain more in-depth answers, there should a measurement/research about the activities of gluconeogenesis, such as about the plasma lipid profiles and glucose liver functions (which were not carried out in this research).

\section{Acknowledgment}

The authors would like to thank the Vocational Faculty of Universitas Airlangga for supporting the research.

\section{Funding}

This research is funded by the Research and Technology and Directorate General of Higher Education Ministry, Republic Indonesia.

\section{References}

[1] Born M. dan Wolf E, 2003, Principles of Optic, Electromagnetic Theory of Propagation Interferences and diffraction of light, Cambridge University Press

[2] Dunlop, J. dan Butera J. A, 2006, Ligands targeting the excitatory amino acid transporters (EAATs). Curr. Top. Med. Chem., 2006. 6(17): p. 1897-1906.

[3] Jiang, F., Li, C.G., Rand, M.J., 1997. Mechanisms of electrical field stimulation-induced vasodilatation in the guinea-pig basilar artery: the role of endothelium. Journal of Autonomic Pharmacology 17: 71-76.

[4] Kakorin S, dan Neumann E., 2002, Electrooptical relaxation spectrometry of membrane electroporation in lipid vesicles, A: Physicochemical and Engineering Aspects 209

[5] Parson, W., 2006. Modern Optical Spectroscopy. University of Washington, Springer Berlin Heidelberg, New York, USA.

[6] Pavlin M., Kanduser M., Rebers M., Pucihar G., Hart F. X., Magjarevic R., dan Miklavc D., 2005, Effect of Cell Electroporation on the Conductivity of a Cell Suspension, Biophysical Journal Volume 88 June 2005 4378-4390

[7] Saleh B. E. A., Teich M.C., 2007, Fundamentals of Photonics, John Wiley \& Sons, Inc, Hoboken New Jersey, Canada 